\title{
Morphological Variations and Laterality of Bony Projections on Cerebral Aspect of Petrous Temporal Bone: Its Anatomical and Surgical Relevance
}

Results: Most of the projections seen were bilateral, 168 (64.61\%), however, most of the projections were seen on right side $146(56.15 \%)$. As per their location the projections were either located along superior border, 31 (11.92\%) only in relation to sulcus for superior petrosal sinus or along posterior border, 91 (35.00\%) in relation to sulcus for sigmoid sinus or jugular foramen. Majority of specimens ( $n=138,53.08 \%$ ) showed occurrence of projections along both specified sites.

Conclusion: Presence of majority of projections bilaterally suggests a symmetrical pattern which might be influenced genetically during development or due to environmental/ external factors. A profound knowledge of various projections on postero-superior surface of petrous temporal bone is required by clinicians and surgeons for management and to avoid their misinterpretation as pathological ones. These findings have a relevance in understanding functional and evolutionary anatomy, and to provide individual variations. \begin{abstract}
dural venous sinus. Images were taken and the data was tabulated with percentage incidence of individual observation.

ABSTRACT aspect of petrous temporal bone especially in relation to evaluating and differentiating normal from abnormal.

Aim: This study was aimed to observe the incidence and vation of various bony projections relating to sulcus temporal bone.

Materials and Methods: The study was conducted on 260 skulls (out of which 20 were obtained from cadavers during dissection and 240 were dry skull) in the Department of Anatomy, ANIIMS from April 2016 to December 2019. The cerebral aspect of petrous temporal bone was observed for presence and localisation of projections. They were classified according to their size (small- $<1 \mathrm{~mm}$, medium$1-3 \mathrm{~mm}$, large->3 mm), location, laterality and its relation to sulcus for
\end{abstract}

\section{INTRODUCTION}

Non-metrical variants are said to be occurring because of normal developmental process and inherited by a dominant gene pattern. Such variants are constant in a particular race and thus, can help in differentiating population group by mapping out 'iso-incidence lines. Non-metrical variants in human skull have been documented earlier [1]

The petrous part of temporal bone is wedged between the sphenoid and occipital bones in the base of skull. It is inclined superiorly and anteromedially, and has a base, apex, three surfaces and three borders. The base of petrous part of temporal bone lies on the base of the skull. The apex, blunt and irregular contains the anterior opening of the carotid canal and limits the foramen lacerum posterolaterally. The anterior surface contributes to the floor of the middle cranial fossa and is continuous with the cerebral surface of the squamous part. The inferior surface is rough and irregular and is part of the external surface of the skull base [2].

Dural venous sinuses namely, sigmoid sinus, superior petrosal sinus and inferior petrosal sinus have an intricate relation with petrous part of temporal bone. The posterior surface of petrous temporal bone contributes to floor of posterior cranial fossa and demarcated from anterior surface by superior border where sulcus or superior petrosal sinus is located. The sigmoid sinus is related to its posteroinferior border on cerebral aspect. Sigmoid sinus is a continuation of transverse sinus which starts at the internal occipital protuberance. On each side, transverse sinus passes anterolaterally to the lateral part of petrous temporal bone where it continues as sigmoid sinus.
The superior petrosal sinus communicates with sigmoid sinus at lateral most end of superior border of petrous temporal bone. On each side, the sigmoid sinus curves inferomedially in a deep sulcus on the mastoid part of temporal bone, crosses the jugular process of the occipital bone and then turns anteriorly to continue as the superior bulb of the internal jugular vein in the posterior part of the jugular foramen. Each sigmoid sinus communicates with the vein of the pericranium by means of the mastoid and condylar emissary veins [3].

Temporal bone is very crucial from surgeon's point of view. Many surgical procedures such as mastoidectomy, procedures on the sigmoid sinus and transverse sinus thrombosis and neurosurgeries in temporal region have emphasised the importance of temporal bone and sigmoid sinus variations and their adjacent relationship. Anatomic variations are frequent in the temporal bone with respect to the shape and size of the sigmoid sinus [4].

The skull protects the brain from external injury and isolates the intracranial blood vessels and acts as a buffer along with the meninges and Cerebrospinal Fluid. Anatomists have been studying the variant bony projections, ossicles, foramina, notches and ridges found in skull. These bony projections can be encountered during surgical procedures in clival and petrous regions by a neurosurgeon or while ligating the sigmoid sinus [5].

This study was planned to study the bony projections on cerebral aspect of petrous part of temporal bone to illustrate different variations of these projections. The projections were observed for 
their location, size, shape, pattern, relation, orientation and area covering the sulci for venous sinus.

\section{MATERIALS AND METHODS}

This was an observational study conducted during the period from April 2016 to December 2019. Two hundred and forty (240) dry unsexed adult human skulls of Indian origin were collected from the Department of Anatomy, from bone bank of ANIIMS. The skulls were examined for the presence of bony projections on the cerebral aspect of petrous part of temporal bone. Deformed and damaged skulls with any pathology were excluded from the study. Specified feature of each projection were documented. The incidence, side and laterality of these bony projections were noted. In addition, 20 cadavers were dissected to observe the bony projections.

The assessment of size of each projection was made by classifying it according to its approximate size into: small $<1 \mathrm{~mm}$, medium from 1 to $3 \mathrm{~mm}$, and large $>3 \mathrm{~mm}$ [6]. Other bony features along the superior border were also observed. The findings were photographed and tabulated.

From current study, variable features were noted under following points:

i. Laterality and side of bony projections

ii. Size of bony projection- as small, medium or large

iii. Shape of each projection- whether pinhead shape, small crest like, pointed spine like, serrated, winged or wedged or plate like. However, the individual incidence of each shape was not incorporated in current study.

iv. Relation of projection with respect to sulci for superior petrosal sinus and sigmoid venous sinus.

The projections were classified into two types:

A) Superior projections-Located over superior border in relation to sulcus for superior petrosal sinus.

B) Posterior projections-Those emerging from posterior border on cerebral aspect of petrous temporal bone. They were directed either in relation to jugular foramen or overlying anterior border of sulcus for sigmoid venous sinus.

\section{RESULTS}

The results were obtained in total 260 skulls (240 dry skulls and 20 cadaveric skulls).

The results of laterality and size of all projections collectively have been represented in [Table/Fig-1,2] respectively. [Table/Fig-3] is showing unilateral projections and [Table/Fig-4] is showing bilateral projections of small [Table/Fig-3a,4a], medium [Table/Fig-3b,4b] and large [Table/Fig-3c,4c] sizes each.

\begin{tabular}{|c|c|c|}
\hline Position & Number of cases $(\mathrm{N})$ & Percentage (\%) \\
\hline \multicolumn{3}{|l|}{ Laterality } \\
\hline Unilateral & 92 & 35.38 \\
\hline Bilateral & 168 & 64.61 \\
\hline Total & 260 & 100 \\
\hline \multicolumn{3}{|l|}{ Side } \\
\hline Left & 114 & 43.84 \\
\hline Right & 146 & 56.15 \\
\hline Total & 260 & 100 \\
\hline
\end{tabular}

\begin{tabular}{|l|c|c|c|}
\hline Size & Right N (\%) & Left N (\%) & Total N (\%) \\
\hline Small & $113(77.39)$ & $80(70.17)$ & $193(74.23)$ \\
\hline Medium & $25(36.5)$ & $27(30.78)$ & $52(20)$ \\
\hline Large & $8(11.68)$ & $7(7.98)$ & $15(5.76)$ \\
\hline Total & $146(56.15)$ & $114(43.84)$ & $260(100)$ \\
\hline
\end{tabular}
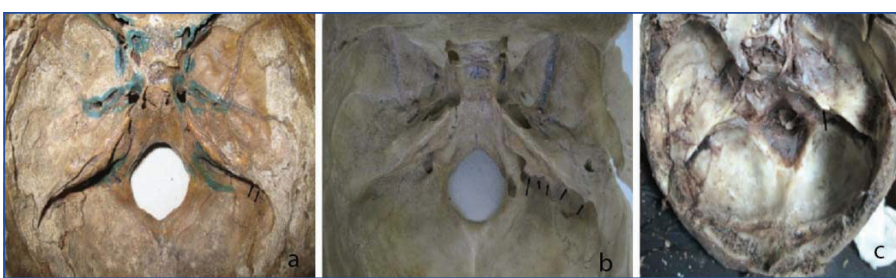

[Table/Fig-3]: Unilateral temporal bony projections in different size; a) Unilateralsmall projection; b) Unilateral-medium projection; c) Unilateral-large projection.
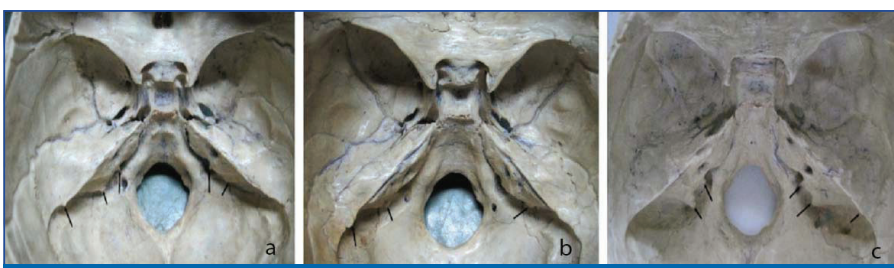

[Table/Fig-4]: Showing bilateral temporal bony projections in different size; a) Bilateral-small projection; b) Bilateral-medium projection; c) Bilateral-large projection.

It was observed that most of the projections were either located along superior border in relation to sulcus for superior petrosal sinus or they were located in relation to posteroinferior border along suture with occipital bone in relation to sulcus for sigmoid venous sinus.

It was observed that symmetrical pattern was more common rather than their asymmetrical occurrence. It signifies that the projections although variable in size, shape, form and position were more often found on both sides. Some of the projections showing bilateral occurrence were like mirror image of each other.

The results correlating the incidence of their position and laterality pattern of two types of projections have been shown in [Table/Fig-5,6].

\begin{tabular}{|l|c|c|}
\hline Location & $\begin{array}{c}\text { Number of cases } \\
\text { (N) }\end{array}$ & $\begin{array}{c}\text { Percentage } \\
\text { (\%) }\end{array}$ \\
\hline Projections at superior border only & 31 & 11.92 \\
\hline Projections at posteroinferior border only & 91 & 35 \\
\hline $\begin{array}{l}\text { Projection occurring both at Superior } \\
\text { Border and Posteroinferior border }\end{array}$ & 138 & 53.08 \\
\hline [Table/Fig-5]: Percentage incidence of projections as per its location. N=260
\end{tabular}

\begin{tabular}{|l|c|c|c|}
\hline Location of projection & $\begin{array}{c}\text { Unilateral } \\
\text { N (\%) }\end{array}$ & $\begin{array}{c}\text { Bilateral } \\
\text { N (\%) }\end{array}$ & $\begin{array}{c}\text { Total } \\
\text { N (\%) }\end{array}$ \\
\hline $\begin{array}{l}\text { Projections at Superior Border (in relation } \\
\text { to sulcus for superior petrosal sinus) }\end{array}$ & $40(48.78)$ & $42(51.22 \%)$ & $82(31.54)$ \\
\hline $\begin{array}{l}\text { Projections at posteroinferior border (in } \\
\text { relation to sulcus for sigmoid sinus and } \\
\text { jugular foramen) }\end{array}$ & $52(29.21)$ & $126(70.79)$ & $178(68.46)$ \\
\hline Total & $92(35.38)$ & $168(64.61)$ & $260(100)$ \\
\hline
\end{tabular}

Unilateral and bilateral projections over superior border only are shown in [Table/Fig-7]. Unilateral and bilateral projection in relation to sigmoid sulcus and jugular foramen only have been depicted in [Table/Fig-8]. The samples studied showed projections occurring both at superior border and posteroinferior border on cerebral aspect [Table/Fig-9a-c].

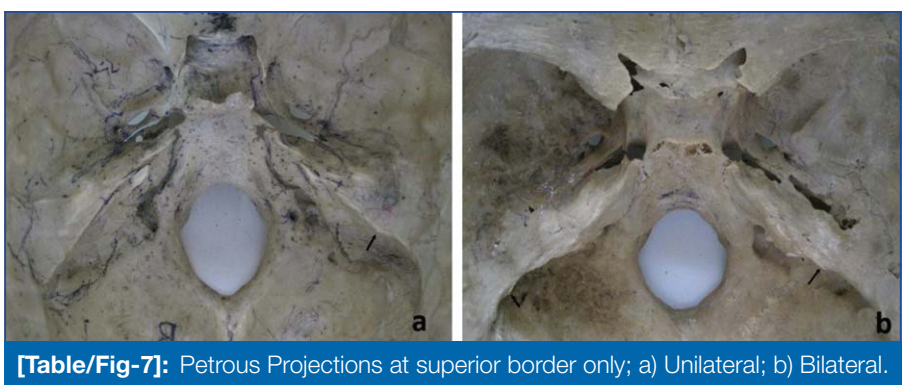



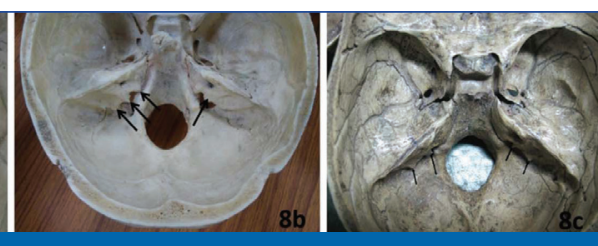

[Table/Fig-8]: Petrou
b) Bilateral; c) Bilatera.
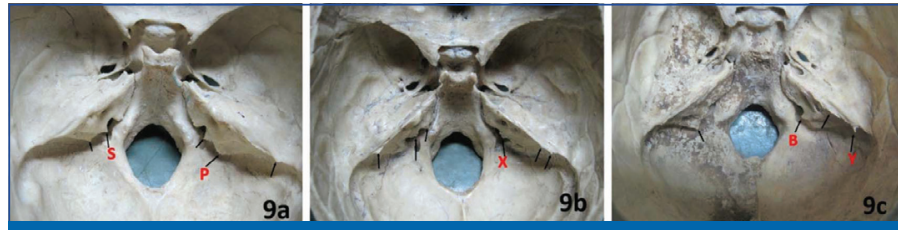

[Table/Fig-9]: Petrous projections both at Superior border and Posteroinferior border; a) Plates (Arrow 'P') and spine (Arrow 'S') like; b) Serrated/tooth like (Arrow 'X'); c) Arrow a) Plates (Arrow ' $P$ ') and spine (Arrow 'S') like; b) Serrated/tooth like (Arrow ' $X$ ');
' $Y$ ' is denoting a process, Arrow ' $B$ ' is representing bridge over jugular formen.

\section{DISCUSSION}

Variability in shape and form of skull are said to be accounted by race, physiology and any pathology. Various factors influencing bone remodelling via bone resorption or deposition could reform the architecture of bony tissue. The variable projections observed on petrous temporal bone can occur as a result of external pressure or tension which affects bone development. The factors responsible for pressure or tension along the site of these projections may be hemodynamic in nature due to dural venous sinuses or traction/ pull due to attachment of dura mater at these sites. There is scant information regarding various projections on postero-superior surface of petrous temporal bone especially in relation to dural venous sinuses or at the site of dural attachment. Detailed anatomical knowledge is required for evaluating and differentiating normal and abnormal projections in this region. This study adds on to the said purpose and the findings of earlier literature.

A study done by Monsef AA on Egyptian skulls documented presence of these projections on $46.15 \%$ [6]. It was documented that bony projections occurred in relation to sigmoid sinus groove and superior petrosal sinus. They were either single, double or multiple. They also observed projections as either simple elevation or sharp spines or shelf like. Such variations were also observed in current study wherein projections seen were of variable shape as shown in [Table/ Fig-9]. Some of the projections at postero-inferior border which were showing serrations at their summit were observed in relation to sulcus for sigmoid sinus [Table/Fig-9b]. This study on Egyptian skulls did not classify projections occurring at superior border as different from those occurring at inferior border. The projection occurring at inferior border were not accounted for, which were included in present study and further classified. The findings related with laterality and size were comparable [Table/Fig-10]. posterior border [Table/Fig-9]. Their size showed marked variation on both sides. In current study, as mentioned earlier their individual incidence was not documented. There was no correlation between size of the projection with the depth of sulcus for sigmoid sinus.

On comparison, the projections were more often seen at posterior border (68.46\%) as compared to superior border (31.54\%). A large percentage of projections occurring at posterior border had a symmetrical pattern (70.79\%). Earlier study by Singh P et al., documented bilateral symmetry in the projection along sigmoid sinus sulcus in $32.9 \%$ cases [7]. In present study, asymmetrical pattern was seen in $35.38 \%$. The symmetrical pattern was more profound in projections seen along postero-inferior border (70.79\%) than along superior border (48.78\%) [Table/Fig-6]. Strong attachments of retinaculum bands of dura mater along the lips of sigmoid sinus were said to be responsible for these bony outcrops. However, no bridges were accounted in present study. However, some bony projections emanating from posterior border partially covered cerebral aspect of jugular foramen [Table/Fig-8c,9c]. This finding should be known to surgeon while doing Petro-Occipital Trans Sigmoid (POTS) approach for jugular foramen lesions. Earlier Computed Tomography (CT) findings have shown normal bony projections and pathological bone involvement at the site of jugular foramen $[7,8]$.

Another literature by Choudhary $\mathrm{R}$ et al., on 636 temporal bone regions of Indian skull, $15.9 \%$ projections were observed at upper part of groove for sigmoid sulcus [5]. Overall percentage occurrence of projections was unilateral in $72.3 \%$ and bilateral in $27.7 \%$. Similar to current study a large number of projections were small (69.3\%) in size. The comparative findings have been depicted in [Table/Fig-10].

A similar study by Afroz A et al., (2002) on 100 temporal bones showed unilateral projections in $27.3 \%$ cases only which was different from current study and previous studies [9]. The laterality pattern showed equal incidence. They described that in $77.3 \%$ cases, the superior petrosal sulcus was superior to projections. In current study also, all the projections seen along superior border had similar relation with superior petrosal sulcus.

From the result of present study, it appeared that certain projections seem to exist at a constant location more often such as those occurring at the superior border of petrous temporal bone. The present findings also record that petrous projections are unique in their site of occurrence which is not documented in detail in earlier literature. Cause for symmetrical presence in some and asymmetrical occurrence in others needs to be further elucidated. Various factors including environmental, genetic and dietary have been documented which influence laterality differences in human skull. Some of the morphological variations have been accounted to agricultural transition also [10]. In addition, genetic factors controlling lateralisation during embryonic development may have a role in determining asymmetry in occurrence of these projections.

\begin{tabular}{|c|c|c|c|c|c|c|c|c|}
\hline \multirow[b]{3}{*}{ Author and Year of study } & \multirow[b]{3}{*}{ Sample size and race } & \multicolumn{7}{|c|}{ Incidence of variable observed } \\
\hline & & \multicolumn{2}{|c|}{ Position } & \multicolumn{3}{|c|}{ Size } & \multicolumn{2}{|c|}{ Location } \\
\hline & & Unilateral & Bilateral & Small & Medium & Large & Right & Left \\
\hline Afroz A et al (2002) [9] & $\begin{array}{c}\mathrm{N}=50 \text { (100 temporal bones } \\
\text { (Bangladesh) }\end{array}$ & $12(27.31 \%)$ & 32 (72.7\%) & $26(59.1 \%)$ & $15(34.1 \%)$ & $3(6.8 \%)$ & 22 & 22 \\
\hline Choudhary R et al (1998) [5] & $\mathrm{N}=318$ skulls (India) & $73(72.3 \%)$ & $28(27.7 \%)$ & 70 (69.3\%) & $16(15.8 \%)$ & 15 (14.8\%) & -- & -- \\
\hline Monsef AA (2006) [6] & N=130 skulls (Egyptian) & $40(66.6 \%)$ & $20(33.3 \%)$ & $40(66.66 \%)$ & $15(25 \%)$ & 5 (8.33\%) & $25(41.66 \%)$ & 35 (58.33\%) \\
\hline Present study (2020) & $\mathrm{N}=260$ (Indian) & 92 (35.38\%) & $68(64.61 \%)$ & $193(74.23 \%)$ & $52(20 \%)$ & 15 (5.76\%) & 146 (56.15\%) & $114(43.84 \%)$ \\
\hline
\end{tabular}

In another study by Singh P et al., (2004), the projections occurring at posterior border only on cerebral aspect of petrous temporal bone were documented [7]. They were notified on anterior lip of sigmoid sulcus which were said to be emanating from posterior border of petrous temporal bone. Their shape was further classified as crests (42.9\%), Plates (22.3\%) and bridges (13.5\%). Such variable forms as crests, plates, spines and serrations/toothed were seen along

\section{Limitation(s)}

In present study, morphometric localisation of these projections from fixed anatomical landmarks were not done which may be helpful in management of surgical procedures in this region. Also, it was not a comparative age-based study which might provide time of appearance of such projections and hence can be a tool for age assessment as well. 


\section{CONCLUSION(S)}

The detailed anatomy of bony projections studied in present study will provide new appreciation and understanding of petrous temporal bone anatomy along with its functional correlation with surrounding structures. Such findings could be beneficial in understanding the origin of any human race synapomorphies. These findings may aid in understanding functional and evolutionary anatomy also. Measurement and observation of such landmarks in this region are important to elucidate various individual variations.

Contribution of authors: SP and VA were actively involved in observation and photographing of bones. Both author were affiliated to ANIMS when study was conducted. MS helped in analysis, did proof reading and drafting of manuscript.

\section{REFERENCES}

[1] Berry AC, Berry RJ. Epigenetic variation in human cranium. J Anat. 1967;101 (Pt. 2):361-79.

[2] Standring S. Gray's Anatomy, The Anatomical Basis of Clinical Practice. London: Churchill Livingstone Elsevier; 41 ${ }^{\text {st }}$ edition. 2016; 826-836.
[3] Gerald F, Folan J. Clinical neuroanatomy and related neuroscience $5^{\text {th }}$ ed. W.B. Saunders. 2004;33-35.

[4] Kayahoglu G, Govsa F, Erturk M, Arisoy Y, Varol T. An anatomical study of the sigmoid sulcus and related structures. Surg Radiol Anat.1996;18:289-94. https://doi.org/10.1007/BF01627607.

[5] Choudhry R, Tuli A, Choudhry S, Kakar S, Raheja S. Anatomical description and frequencies of bony projections on the cerebral aspect of the petromastoid part of the temporal bone in dry adult human skulls. Acta Anat. 1998;162(1):56-60

[6] Monsef AA. Bony variations in the cerebral aspect of petromastoid part of adult egyptian temporal bones. Bull Alex Fac Med. 2006;42(2):508-09.

[7] Singh P, Tuli A, Choudhary R, Dhall U, Makwane UK. Morphology and imaging of bony projections on sigmoid sulcus with clinical implications. Surg Radiol Anat. 2004;26(1):46-50.

[8] Mazzoni A. The petro-occipital trans-sigmoid approach for lesions of the jugula foramen. Skull Base. 2009;19(1):48-56.

[9] Afroz A, Hossain SMA, Rahman L. Incidence and morphometry of bony projections on the cerebral aspect of the petromastoid part of temporal bone in adult human skulls. Pak J Med Sci. 2002;18(3):235-38.

[10] Katz CD, Grote MN, Weaver TD. Changes in human skull morphology across the agricultural transition are consistent with softer diets in preindustrial farming groups. Proceedings of national academy of sciences of the United States of America/PNAS. 2017;114(34):9050-55.

\section{PARTICULARS OF CONTRIBUTORS:}

1. Associate Professor, Department of Anatomy, GAIMS, Bhuj, Gujarat, India.

2. Assistant Professor, Department of Anatomy, BSAMCH, Delhi, India

3. Associate Professor, Department of Anatomy, JNMC, DMIMS, Wardha, Maharashtra, India

\section{NAME, ADDRESS, E-MAIL ID OF THE CORRESPONDING AUTHOR:}

Dr. Sachin Patil,

Associate Professor, Department of Anatomy, GAIMS, G K General Hospital Campus,

Opposite Lotus Colony, Bhuj, Gujarat, India.

E-mail: drsachin6880@gmail.com
PLAGIARISM CHECKING METHODS: [Jain Het al.]

- Plagiarism X-checker: Sep 23, 2020

- Manual Googling: Dec 02, 2020

- iThenticate Software: Feb 22, 2021 (8\%)

\section{AUTHOR DECLARATION:}

- Financial or Other Competing Interests: None

- Was Ethics Committee Approval obtained for this study? NA

- Was informed consent obtained from the subjects involved in the study? NA

- For any images presented appropriate consent has been obtained from the subjects.

Date of Submission: Sep 22, 2020

ETYMOLOGY: Author Origin

Date of Peer Review: Nov 09, 2020

Date of Acceptance: Jan 06, 2021

Date of Publishing: Apr 01, 2021 